\title{
Comments on: Randomized Controlled Trial Comparing Everting Sutures with a Lateral Tarsal Strip for Involutional Lower Eyelid Entropion
}

\author{
Aaron Jamison (D) · David F. Gilmour · John Buchan
}

Received: November 2, 2019 / Published online: February 24, 2020

(C) The Author(s) 2020

Keywords: Entropion; Everting sutures; Eyelid; Involutional; Jones; Jones retractor plication; Lateral tarsal strip; Oculoplastic; Randomised controlled trial

\section{Key Summary Points}

"Combined" entropion surgery (lateral tarsal strip procedure combined with either everting sutures or Jones retractor plication) offers good long-term success.

Researchers planning a randomised control trial should consider excluding participants at increased risk of death during the follow-up period, while clinicians discussing surgical options with their patients may wish to consider life expectancy when choosing between "combined" entropion surgery and more straightforward everting sutures.

Enhanced digital features To view enhanced digital features for this article go to https://doi.org/10.6084/ m9.figshare.11536995.

\section{A. Jamison $(\bowtie)$ - D. F. Gilmour}

Tennent Institute of Ophthalmology, Glasgow, UK

e-mail: Aaronjamison@gmail.com

D. F. Gilmour · J. Buchan

Leeds Teaching Hospitals NHS Trust, Leeds, UK
Dear Sir,

We read with great interest the recent article by Nakos et al. that showed the lateral tarsal strip (LTS) to have superior surgical success rates and reduction of symptoms than Quickert everting sutures (ES) in the repair of primary involutional lower eyelid entropion after one year [1]. We wish to complement their findings with those of our own Yorkshire Entropion Study (not previously published)-a randomised controlled trial with long-term (12-year) follow-up in which we attempt to compare two techniques for the surgical correction of involutional entropion.

Nakos et al. discuss the two key causative factors in primary involutional lower eyelid entropion (horizontal lid laxity, mainly due to senile lateral canthal tendon elongation or dehiscence, and vertical laxity, due to detachment of the inferior retractors from the tarsus) and have designed a study that evaluates the comparative success rates of repairs that address either the horizontal (LTS) or vertical (ES) component. In the Yorkshire Entropion Study we have compared two different "combined" procedures that address both components simultaneously-LTS plus ES vs. LTS plus Jones retractor plication. The primary outcome measure was success (no need for further surgery) vs. failure (need for further surgery) at two-year follow-up, although data collection was possible 
beyond this time point, the results of which are presented here.

Our study was granted ethical approval by the Leeds Teaching Hospital NHS Trust research ethics committee. This study adhered to the tenets of the Declaration of Helsinki, and informed consent was obtained from all study participants. Ninety-nine participants (mean age $=77$ years, range 60-92) were recruited into the study between October 2006 and December 2007 and randomised into the LTS + ES group $(n=53)$ or the LTS + Jones group $(n=46)$. Of the 99 recruited participants, 26 were removed because of participant withdrawal or complete non-attendance of follow-up appointments. We attempted to obtain 12-year follow-up, and a further 14 participants were subsequently removed as their case records were either destroyed or were not traceable by the time of the study's conclusion. Of the remaining 59 participants included in the study, 32 were in the LTS + ES group and 27 were in the LTS + Jones group. Post-operative follow-up data beyond three months are available for only 31 participants (16 LTS + ES, 15 LTS + Jones).

There were no cases of undercorrection in either group at first follow-up. Overcorrection occurred in no participants in the LTS + ES group and in one participant $(3.7 \%)$ in the LTS + Jones group. Recurrence of entropion occurred in no LTS + Jones participants during the follow-up period, but did occur in two patients in the LTS + ES group (6.3\%). The first underwent LTS + ES in 2007 and required further entropion surgery in 2011 (four years postoperatively). The second underwent LTS + ES in 2007 and re-presented with entropion in 2017 (ten years post-operatively) although they have not opted for further surgery to date.

The main limitation of our study is the large source of potential bias created by the number of participants $(n=40)$ removed from the study. Furthermore, if we limit our analysis to only the 31 patients with follow-up data beyond three months the study becomes underpowered, such that comparative analysis would not achieve statistical significance. Finally, one-year follow-up is sufficient to compare the efficacy of two contrasting procedures such as ES vs. LTS, but is too short to identify the more subtle differences between more similar procedures such as LTS + ES vs. LTS + Jones, given their excellent success rates.

We wish to use our randomised trial to highlight the difficulties of performing studies that require a longer follow-up period in an elderly cohort with an increased mortality rate. Nonetheless, our findings suggest that "combined" entropion surgery (either LTS + ES or LTS + Jones) offers good long-term success. Researchers planning a randomised control trial should consider excluding participants at increased risk of death during the follow-up period, while clinicians discussing surgical options with their patients may wish to consider life expectancy when choosing between "combined" entropion surgery and more straightforward everting sutures.

\section{ACKNOWLEDGEMENTS}

Funding. No funding or sponsorship was received for this study or publication of this article.

Authorship. All named authors meet the International Committee of Medical Journal Editors (ICMJE) criteria for authorship for this article, take responsibility for the integrity of the work as a whole, and have given their approval for this version to be published.

Disclosures. The authors (Aaron Jamison, David F Gilmour and John Buchan) report no personal, financial, commercial, or academic conflicts of interest.

Compliance with Ethics Guidelines. This article is based on previously conducted studies and does not contain any studies with human participants or animals performed by any of the authors.

Open Access. This article is licensed under a Creative Commons Attribution-NonCommercial 4.0 International License, which permits any non-commercial use, sharing, adaptation, distribution and reproduction in any medium 
or format, as long as you give appropriate credit to the original author(s) and the source, provide a link to the Creative Commons licence, and indicate if changes were made. The images or other third party material in this article are included in the article's Creative Commons licence, unless indicated otherwise in a credit line to the material. If material is not included in the article's Creative Commons licence and your intended use is not permitted by statutory regulation or exceeds the permitted use, you will need to obtain permission directly from the copyright holder. To view a copy of this licence, visit http://creativecommons.org/licenses/by$\mathrm{nc} / 4.0 /$.

\section{REFERENCE}

1. Nakos EA, Boboridis KG, Kakavouti-Doudou AA, Almaliotis DD, Sioulis CE, Karampatakis VE. Randomized controlled trial comparing everting sutures with a lateral tarsal strip for involutional lower eyelid entropion. Ophthamol Ther. 2019;8:397-406. 